University of Nebraska - Lincoln

DigitalCommons@University of Nebraska - Lincoln

October 1981

\title{
Piezomodulation spectroscopy of molecular crystals. I. Methods and principles
}

\author{
J. Merski \\ University of Nebraska - Lincoln \\ Craig J. Eckhardt \\ University of Nebraska - Lincoln, ceckhardt1@unl.edu
}

Follow this and additional works at: https://digitalcommons.unl.edu/chemistryeckhardt

Part of the Chemistry Commons

Merski, J. and Eckhardt, Craig J., "Piezomodulation spectroscopy of molecular crystals. I. Methods and principles" (1981). Craig J. Eckhardt Publications. 32.

https://digitalcommons.unl.edu/chemistryeckhardt/32

This Article is brought to you for free and open access by the Published Research - Department of Chemistry at DigitalCommons@University of Nebraska - Lincoln. It has been accepted for inclusion in Craig J. Eckhardt Publications by an authorized administrator of DigitalCommons@University of Nebraska - Lincoln. 


\title{
Piezomodulation spectroscopy of molecular crystals." ${ }^{\text {a) }}$. Methods and principles
}

\author{
J. Merski ${ }^{\text {b) }}$ and C. J. Eckhardt ${ }^{c)}$ \\ Department of Chemistry, University of Nebraska, Lincoln, Nebraska 68588 \\ (Received 9 October 1980; accepted 3 March 1981)

\begin{abstract}
The effects of piezomodulation on the spectra (particularly reflectivity spectra) of molecular crystals for the strong, weak, and no coupling regimes are considered. The stress or strain modulation of the crystal is shown to affect the dispersion energy and exciton resonance interaction. The experimental approach is outlined. Methods of data analysis are described for obtaining differential changes in the real and imaginary parts of the dielectric function, for determining deformation potentials for vibronic transitions, and for applying the Seraphin coefficients. Previous microscopic theory for interaction of excitons with static lattice deformations is adapted for the modulated case. Application of classical dipole theory to model one and two vibronic level systems yields expressions for calculation of the piezomodulated reflection spectrum. Relative contributions to the spectrum are determined for a model polariton stop band by consideration of piezomodulation of the exciton energies, plasma frequency, oscillator strengths, and background dielectric function. Applications of
\end{abstract} \\ piezomodulation spectroscopy to investigations of molecular and ionic crystals are discussed.
}

\section{INTRODUCTION}

Pressure effects are commonly observed in spectroscopy. The solid state manifestation is found in lattice strain and its effect on the spectra of the crystals can lead to extreme perturbation of the bands. In the electronic spectra of molecular crystals the role of strain is of importance but has been little studied. Indeed, strain is often invoked to explain frequency shifts or intensity changes but little quantitative information is available. High pressure studies ${ }^{1}$ have been extremely useful in understanding the role of pressure in condensed media but in this regime one is usually dealing with plastically deformed material and the effects of the specific stress-induced perturbations of the crystal states cannot be easily deduced. Such studies, therefore, investigate either energetics quite high up in the potential energy surface of the crystal or, more commonly, those of a surface significantly altered from that at standard pressure.

Even for relatively low oscillator strength exciton transitions, rapid extinction of incident light necessitates the use of thin crystals $(1-5 \mu)$. The latter are often mounted on a quartz substrate and cooled for low temperature investigation of the absorption spectrum. The differential thermal contractions of the crystalsubstrate system invariably result in large induced strains in the sample. The resulting distortions in the exciton absorption spectrum provided the first semiquantitative data on exciton-strain coupling. Colson ${ }^{2}$ enumerated the phenomenological consequences of lattice deformation on the ${ }^{1} B_{2 u}-{ }^{1} A_{1 \xi}$ transitions in benzene: strain produced transition energy shifts, absorption band broadening, a pronounced change in the factor group splitting, and an induced polarization in one of the absorption components. These effects were not in-

\footnotetext{
a Research supported by the Solid State Chemistry Program, Division of Materials Research of the National Science Foundation (DMR 79-08-759).

b) Part of a dissertation submitted to the University of Nebraska in partial requirement for the Ph. D. degree.

c)John Simon Guggenheim Fellow, 1979-80.
}

vestigated further in any detail but it was speculated that the changes could be attributed to a strain-induced modification of the van der Waals and exciton resonance interactions. It was also suggested that stresses applied in a more controlled manner along different crystallographic axes would be helpful in elucidating spectral features and making transition moment polarization assignments. Somewhat later, Hanson ${ }^{3}$ suggested similar beneficial uses of uniaxial stress in resonance pair spectroscopy. Despite the apparent potential of stress experiments, no experimental effort was mounted to exploit the obvious possibilities. Recent work ${ }^{4}$ has shown that the strain generated in crystal mounting strongly perturbs the $S_{1}$ spectrum of anthracene, thus confirming earlier speculation that the great variance in reported Davydov splittings for this system were due to strain. 5

The first detailed investigation of strain effects in a molecular crystal was reported for anthracene by Morris, Rice, and Martin. ${ }^{8}$ Antireflection resonances attributed to exciton states bound to lattice defects were observed upon cooling the crystal from 77 to $4 \mathrm{~K}$. Similar phenomena were later observed by Morris and Sceats ${ }^{7}$ and attributed to a high density of submicroscopic voids in the crystal. They later reported for the $S_{1}$ system of anthracene significant exciton shifts, band shape distortion, and oscillator strength change due to crystal surface roughness, bulk defects, strain, and lattice contraction upon cooling. ${ }^{8(a),(b)}$ These detailed studies were part of a broader effort concerned with elucidating the features of exciton dynamics, more specifically, polariton-phonon scattering; thus, the study of strain perturbation of excitons was not pursued.

In the scattered collection of published data which could exhibit possible exciton-strain coupling, there is very little unambiguous quantitative information. Reported results often display great variances due to differences in crystal purity, defect concentration, crys tal thickness, mounting procedures, and rates of cooling. Morris and Sceats $^{8}$ reported a mean vibronic ex- 
citon shift for the $(0,0)$ band of the $S_{1}$ anthracene transition of approximately $150 \mathrm{~cm}^{-1}$ upon cooling the crystal from room temperature to $77 \mathrm{~K}$. Part of this shift is due to the temperature dependence of the exciton-phonon coupling and therefore not solely attributable to lattice contraction. The latter may be evaluated from the temperature dependence of the lattice parameters reported by Mason. ${ }^{9}$ The fractional volume change is $3.6 \%$ which, using the volume or bulk isothermal compressibility of anthracene from Bridgman's data, ${ }^{10}$ gives an equivalent pressure of approximately $4 \mathrm{kbar}$. This pressure and the previously reported exciton shift of $150 \mathrm{~cm}^{-1}$ indicate a difference in ground and excited state deformation potentials of approximately $37.5 \mathrm{~cm}^{-1} /$ kbar. This value is in good agreement with the published data from high pressure optical spectroscopy studies of anthracene. ${ }^{1}$ Thus, one has at least an estimate for the exciton strain sensitivity. This figure should probably be taken advisedly in view of the relatively high equivalent pressure of $4 \mathrm{kbar}$. As noted by Kalinowski et al., ${ }^{11}$ approximately the same lattice contraction upon cooling tetracene crystals results in a phase transition. Consequently, even such a relatively low equivalent pressure may not be interpreted, a priori, as corresponding to the linear or elastic stress-strain regime of the crystal. Thus, no reliable low pressure or linear piezo-optic data on organic single crystals exist. In the same vein, there is a similar scarcity of single crystal elastic constant data required for detailed interpretation of stress -optic experiments. Thus, pressure or strain techniques have not been incorportated into single crystal optical spectroscopy of organic compounds. In most instances, strain has been regarded as a nuisance variable rather than a potentially useful perturbation.

There are many experimental difficulties and limitations to high pressure optical spectroscopy. Most high pressure experiments on solids are restricted to room temperature, unpolarized, absorption or luminescence spectroscopy under conditions of quasihydrostatic pressure where, usually, induced intermolecular distance and orientation changes are uncertain. Additionally, induced phase transitions and plastic deformation of the solid are common. Lastly, spectral resolution decreases rapidly with increasing pressure due to band broadening. The alternative to high static pressures is the use of rather small dynamic homogeneous pressures or strains which are within the linear or elastic region of the stress-strain curve for the molecular crystal. Differential piezomodulation techniques accomplish this objective.

An internal or active modulation spectroscopy technique relies on the dynamic perturbation of the sample to induce a change in the optical response function. The latter is detected by a synchronous demodulation of the usually sinusoidal signal. The perturbation may be a mechanical stress, an electric or magnetic field, a temperature change, or some combination of the above. In all cases it is kept small and hence the induced modulation of the optical response function is usually several orders of magnitude below detection system noise levels. Consequently, accurate and reproducible measurement of the signal is experimentally demanding.

The fundamental ideas indigenous to the modulation spectroscopy of solids are not new. Since the advent over a decade ago of sample modulation techniques to solid state spectroscopy, they have remained, until recently, almost exclusively in the domain of solid state physics. Seraphin and Hess ${ }^{12(a)}$ were the first to report the modulated electroreflection of germanium (Franz-Keldysh effect). The reported spectral profile of the electric field-induced fractional reflectivity changes $\Delta R / R$ is highly structured in comparison to the unmodulated reflectivity. It has subsequently become possible to correlate the observed structural features in $\Delta R / R$ with critical points in the interband joint density of states of germanium. In rapid succession, other dynamic sample perturbation techniques were tried with comparable success. Engeler et al., ${ }^{12(b)}$ Gobeli and Kane, ${ }^{12(\mathrm{c})}$ and Garfinkel et al. ${ }^{12(\mathrm{~d})}$ pioneered the area of piezoreflection or strain modulated reflectivity. Batz ${ }^{13(a)}$ was the originator of thermomodulation and Balslev ${ }^{13(b)}$ demonstrated the utility of an external modulation technique, wavelength modulation. The area of research may be surveyed through the reviews of Cardona, ${ }^{14(a)}$ Seraphin, ${ }^{14(b)}$ and that edited by Willardson and Beer. ${ }^{14(c)}$

Common to modulation spectroscopy techniques is the high sensitivity and optical resolution capability associated with differential measurement methods. Any enhancement in resolution favors the possibilities of correlating optical spectral features with specific crystal eigenstates.

Utilization of modulation techniques in the spectroscopy of organic crystals has been meager. Hoch strasser and Noe ${ }^{15}$ reported the Stark modulation of the fluorescence and phosphorescence of phenanthrenedoped biphenyl. Electric field modulation has also been employed by Hanson. ${ }^{3}$ Matsui et al. ${ }^{18}$ investigated the first singlet exciton state of anthracene by thermoreflection techniques which were also used by Perov and Fisher ${ }^{17}$ to probe TTF-TCNQ. A most elegant application of dynamic sample perturbation methods is the microwave-optical double resonance experiment of $\mathrm{El}$ Sayed. ${ }^{18}$ Lastly, modulated piezoreflection of the crystalline charge transfer complex pyromellitic dianhydride-anthracene was first reported by Eckhardt and Merski. ${ }^{19}$

Of the possible modulation perturbations, we have chosen piezomodulation for the study of molecular crystals for the reasons detailed in the following paragraphs. An internal modulation parameter permits direct interaction with the bands of the crystal and may provide a nonderivative behavior not possible with external modulation. At low frequencies $\left(10-10^{4} \mathrm{~Hz}\right)$ the stress waves' wavelengths are on the order of meters and thus the perturbation is homogeneous. Translational symmetry of the lattice is not destroyed as it is in electroreflect ance. In contrast to thermoreflectance, the directions along which the perturbation is applied can be controlled and thus the factor group or site symmetries may be altered. While there is a paucity of data for the com- 
plete elastic tensors of molecular crystals, it is, nevertheless, more abundant than the thermal expansion tensors, nor is the dielectric response known for the driving electric fields used in electroreflectance. By use of Brillouin scattering, the elastic constant tensor may be obtained with relative ease.

The conceptual framework for piezomodulation is also straightforward. In the case of an oriented gas crystal, the oscillation of the energy levels is expected to lead to a purely derivative spectrum due to modulation of the van der Waals energy of the crystal. How ever, as dipole-dipole interactions increase in the crystal this may change. The strains involved are $\sim 10^{-4}$ and thus any effect on the molecular geometry is miniscule. However, for the lattice, changes may be significant and the movement of the dipoles from their static equilibrium positions can be expected to affect the resonance interaction matrix $I(k)$ as well as the site shift $D$. In principle, once the elastic constant tensor (external and internal) is known, the movement of the molecules in the lattice can be determined for an elastic strain or stress of known configuration. Since the piezomodulation involves a compression and an extension of the lattice, the calculation of the spectrum will involve evaluation of a differential dipole lattice sum. In the case of very strong coupling with small effective masses and high exciton densities, the piezomodulation effect can be expected to be large because of the pressure of the exciton gas and the application of a modulated dipole-dipole interaction model may be inadequate.

It is, however, difficult to assess the validity of extrapolating the results of high pressure studies on organics to the 25-50 bar range in which piezomodulation techniques operate. The relatively weak cohesive forces in molecular solids and the disruptive influence of lattice vibrations present an additional concern. The sample strains usually achieved in piezoreflection are $\approx 10^{-4}-10^{-5}$. Considering a typical molecular crystal such as anthracene, these strains correspond to absolute lattice dimensional changes of $\approx 10^{-3}-10^{-4} \AA$. Mason's ${ }^{9} \mathrm{x}$-ray crystallographic data on anthracene indicate that the thermal torsional oscillations of the molecules are $\approx 3^{\circ}$ and that translational displacements are $\approx 0.2 \AA$ at $290 \mathrm{~K}$. Consequently, piezomodulation must successfully compete or interact with excitonphonon coupling if one hopes to generate detectable changes in reflectivity. A wide variety of crystal systems spanning the range of dipole coupling cases from weak to very strong require investigation and such studies are reported in the papers that follow. Of particular interest are those exhibiting very strong coupling, for in these quasimetallic crystals the reflection band is often quite structureless and little gain of resolution is observed on cooling, especially in the multiparticle region. ${ }^{20}$

Determination of the modulation sensitivity of the exciton for the different coupling cases and the effective increase in spectral structure enhancement obtained from the modulated piezoreflection spectrum is required. The spectrum should also be compared with a numerical energy derivative of the unmodulated reflectivity. Comparison of the magnitudes and signs of the exciton shifts ascertained from piezoreflection with the extrapolated high pressure optical results is useful for aralysis. The $\Delta R / R$ data must be Kramers-Kronig transformed to yield the strain-induced changes in the complex dielectric function $\vec{\epsilon}(\omega, \mathbf{k})$. A strain modulation is, in general, expected to result in exciton energy shifts, oscillator strength changes, and exciton bandwidth modulations which are amenable to a phenomenological description of reflection modulation in terms of the basic exciton interactions. The modulated piezoreflection of molecular crystals examined here represents the first assessment of the potential of such a technique to the field.

This paper is structured as follows: In Sec. II a brief discussion of the experimental method of piezomodulation spectroscopy is given. In Sec. II the techniques for data reduction and analysis are presented. The interpretation of piezomodulated reflection spectra is given in Sec. IV for the oriented gas, weak to intermediate coupling, and very strong coupling regimes for molecular crystals. The summary and conclusions are given in Sec. V. The paper itself is followed by three papers dealing with experimental results. The latter treat, in detail, the three coupling cases considered in Sec. IV.

\section{EXPERIMENTAL METHODOLOGY}

While details of the experiment are reported elsewhere, ${ }^{14,19,21}$ a brief sketch of the method will be given for orientation. The stress (strain) is applied by attaching a crystal of approximately $0.2 \mathrm{~mm}$ thickness to a lead zirconate titanate (PZT) ceramic. The ceramic is driven through cycles of extension and compression by an applied alternating voltage which is usually on the order of $1000 \mathrm{~V}$ (peak value). The resulting strains are on the order of $10^{-4}$ to $10^{-5}$. The ceramic may be driven either resonantly or nonresonantly. The latter was the usual method since the motions of the ceramic are easier to analyze. The driving frequencies range from $10^{2}$ to $10^{4} \mathrm{~Hz}$. Since frequency dependence of the piezomodulation spectra has not been observed in this range, it has not been considered in the development in this paper. Its importance appears in piezomodulated emission and photoconductivity experiments.

The crystal is bonded to the ceramic by epoxy and, depending upon whether the crystal is bonded completely to the transducer or between two such transducers in an anvil arrangement, a strain or stress modulation is obtained, respectively. The strain (stress) is measured by a strain gauge and by calculation using the piezoelectric constants of the transducer.

The signal of the normally incident polarized specularly reflected light is detected by a photomultiplier whose photocurrent is measured synchronously with the driving voltage. The phototube is maintained at a constant dc value. Ensuing signals are ensemble averaged and subjected to a confidence level for acceptance. The signal is recorded as a function of photon energy. 
The effects observed are not due to oscillation of the index or extinction ellipsoids about the principal directions since this would give rise to a signal at twice the driving frequency. Such a signal, if present, is significantly less than the noise associated with the experiment.

\section{PIEZOREFLECTION ANALYSIS}

Modulated piezoreflection spectra are directly analyzed through the differential form of Fresnel's equation for normal incidence reflectivity. One may either attempt to establish a rigorous theoretical fit to $\Delta R / R$ based on a crystal dielectric function or extract the strain-induced modulation of the latter by KramersKronig analysis of the piezoreflection spectrum. The merits of the integral transform option are apparent. The strain perturbation of the dielectric function $\Delta \bar{\epsilon}$ may be obtained directly from the primary experimental data $R, \Delta R / R$, and the dispersion relations. The theoretical analysis may be subsequently extended to a consideration of the microscopic contributions to $\Delta \tilde{\epsilon}$. Thus, it is expected that Kramers-Kronig transformation of piezoreflection spectra is necessary for complete analysis. In addition to transform procedures, some form of analysis of the primary data $\Delta R / R$ is also desirable. Recognition of specific contributions to $\Delta R / R$ obtained without recourse to a Kramers-Kronig transform can be expected to yield information which supplements an analysis based on the latter.

\section{A. Seraphin coefficients}

In lieu of a rigorous theoretical fit to the $\Delta R / R$ spectrum, it has been shown that the composition of the latter may be partially ascertained with the aid of

$$
\begin{aligned}
\frac{\Delta R}{R} & =\frac{1}{R}\left(\frac{\partial R}{\partial \epsilon_{r}}\right)_{0_{i}} d \epsilon_{r}+\frac{1}{R}\left(\frac{\partial R}{\partial \epsilon_{i}}\right)_{\epsilon_{r}} d \epsilon_{i}, \\
& =\alpha\left(\epsilon_{r}, \epsilon_{i}\right) \Delta \epsilon_{r}+\beta\left(\epsilon_{r}, \epsilon_{i}\right) \Delta \epsilon_{i},
\end{aligned}
$$

where $\alpha$ and $\beta$ are the Seraphin coefficients, and $\epsilon_{i}$ and $\epsilon_{r}$ are the imaginary and real parts of the dielectric constant, respectively. A knowledge of the spectral profiles of $\alpha$ and $\beta$ has been used in the recognition of specific $\Delta \tilde{\epsilon}$ contributions to $\Delta R / R$, consequently enabling an interpretation of the modulated spectrum. A second method of analyzing the piezoreflection spectrum requires the calculation of the logarithmic frequency derivative of the unmodulated reflection data. The recognition of deviations in $\Delta R / R$ from a strict frequency (or transition energy) derivative may then be explained in terms of two or more concurrently operative band modulation mechanisms.

Seraphin $^{22}$ and Cardona ${ }^{14(a)}$ detail the procedures for analyzing modulation spectroscopy data of metals and semiconductors utilizing the Seraphin coefficients. The unmodulated reflection spectra of these types of materials are generally congested and broadened by the large joint density of states. The recognition of critical point contributions to the density of states function was the principal motivation for applying modulation spectroscopy techniques to metals and semiconductors. In this regard, differential measurement methods proved to be effective in suppressing the noncritical contributions to the optical response function. The latter contributions are responsible for both the diffuse nature of the reflection spectrum and the wellbehaved, slow frequency variations of the Seraphin coefficients $\alpha$ and $\beta$. An approximate interpretation of $\Delta R / R$ spectra benefits immensely from the combined presence of sharply varying critical point structure and the virtual absence of interference from the $\alpha$ and $\beta$ contributions. Furthermore, as Seraphin ${ }^{22}$ points out with specific reference to silicon and germanium, the $\alpha / \beta$ ratio, and consequently the relative weight of $\Delta \epsilon_{r}$ to $\Delta \epsilon_{i}$ in $\Delta R / R$, is usually considerably different from unity in a given spectral region. The modulated optical response in a limited photon energy region is almost entirely attributable to either $\Delta \epsilon_{i}$ or $\Delta \epsilon_{i}$. The characteristically simple spectral behavior of $\alpha$ and $\beta$ for metals and semiconductors facilitates an approximate interpretation of the modulated optical data which is generally not possible for piezomodulation spectra of molecular crystals. The corresponding Seraphin coefficients for the latter are ill-behaved, rapidly varying functions of frequency and regions of predominant response in $\Delta R / R$ from either $\Delta \epsilon_{r}$ or $\Delta \epsilon_{i}$ are narrow or nonexistent. The spectral profiles of $\alpha$ and $\beta$ have been calculated for the crystal systems under consideration and a typical one, that for the $S_{1}$ system in anthracene, is shown in Fig. 1. Because it is not then possible to employ Seraphin coefficient analysis of the piezoreflectance spectra, the latter must be analyzed by comparison with the logarithmic derivative of $R$, i. e., $(1 / R)(\partial R / \partial \omega)_{\Omega_{u}}$. Here, $\omega$ is the photon frequency and $\Omega_{u}$ is the $u$ th exciton frequency.

\section{B. Logarithmic derivatives of reflectivity}

The modulated piezoreflection spectrum of a molecular crystal may, to a first approximation, be expected to mimic the logarithmic frequency derivative of the reflectivity. This anticipated behavior follows from a consideration of the differential form of Fresnel's equation in conjunction with the general formula for the crystal dielectric function appropriate to the particular molecular excitations under investigation. In the case of molecular exciton transitions, the dielectric tensor may be assumed to have the functional form given by

$$
\tilde{\epsilon}(\omega, \mathbf{k})=\epsilon_{0}\left[1+\sum_{u} \frac{\omega_{0}^{2} F_{u}(\hat{\mu} \cdot \hat{\mathbf{e}})^{2}}{\Omega_{u}^{2}-\omega^{2}+i \gamma_{u} \omega}\right],
$$

which obtains for transverse excitons. $\Omega_{u}$ is the $u$ th exciton frequency, $\gamma_{u}$ is a phenomenological damping factor, $\hat{\mu}$ the transition dipole moment direction unit vector, $\hat{e}$ the polarization direction unit vector, $\omega_{0}$ the screened (by $\epsilon_{0}$ ) plasma frequency, and $F_{u}$ the oscillator strength. The $\Delta R / R$ formula for small straininduced changes in $\tilde{\epsilon}$ is given by $\mathrm{Eq}$. (3.1). Examination of the dielectric function reveals five parameters which are potentially sensitive to lattice deformation: $\epsilon_{0}, \gamma_{u}$, $\Omega_{u}^{2}, F_{u}$, and $\omega_{0}^{2}$. The weak coupling or single particle exciton transition energy is susceptible to strain modulation by perturbations of the site shift $D$ and resonance energy $I(k)$. The relatively small transition oscillator strengths in this case obviate the need for considering 


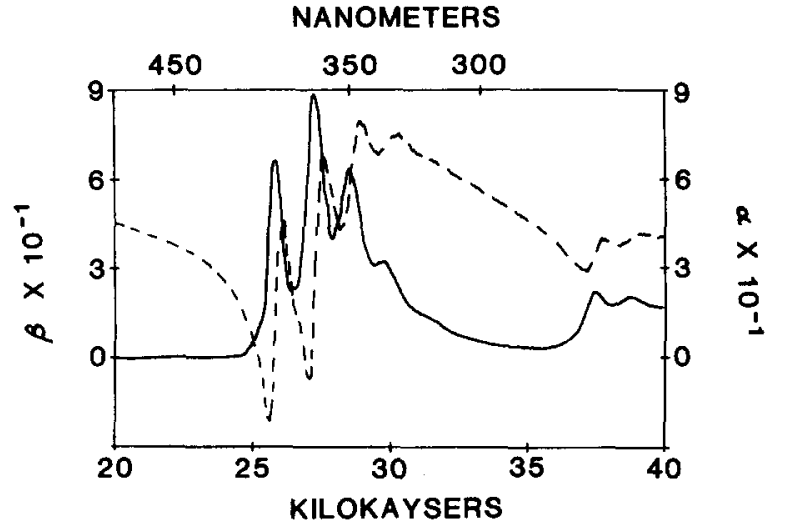

FIG. 1. Seraphin coefficients for the $b$ axis (001) face polarized piezoreflection spectrum for the first singlet system of anthracene at $300 \mathrm{~K} . \alpha(---)$ and $\beta(-)$.

the modulation of either $F_{u}$ or $\omega_{0}^{2}$. The strain sensitivities of $\epsilon_{0}$ and $\gamma_{u}$ are difficult to assess and for the situation under consideration may be assumed to be small. The exciton resonances in the dielectric function are manifested as bands of enhanced specular reflection. Consequently, a transition frequency derivative of $\tilde{\epsilon}$ generates a corresponding derivative line shape in $d R / R$. The logarithmic differential of $R$ with respect to the exciton transition frequency may be written as

$$
\frac{d R}{R} \approx \frac{1}{R}\left(\frac{\partial R}{\partial \Omega_{u}}\right)_{\omega} d \Omega_{u},
$$

which is the assumed approximate form of the measured piezoreflection signal in the vicinity of the $u$ th exciton resonance where $\omega$ represents the photon frequency. The reflectivity may be represented by a Lorentz expression

$$
R \simeq A+\frac{B}{\left(\Omega_{u}-\omega\right)^{2}+\gamma_{u}^{2}} .
$$

Consideration of Eqs. (3.4) and (3.5) yields the following:

$$
\frac{1}{R}\left(\frac{\partial R}{\partial \Omega_{u}}\right)_{\omega}=-\frac{1}{R}\left(\frac{\partial R}{\partial \omega}\right)_{\Omega_{u}} .
$$

In order to facilitate comparison between $d R / R$ and $(1 / R)(\partial R / \partial \omega) \Omega_{u}$ which must be made in analysis of the experimental data, it is useful to clarify several potential sources of confusion.

From expressions (3.4) through (3.6) one may write

$$
\frac{d R}{R} \simeq-\frac{1}{R}\left(\frac{\partial R}{\partial \omega}\right)_{\Omega_{u}} d \Omega_{u},
$$

which respresents the relationship used to ascertain the strain-induced exciton frequency shifts $d \Omega_{u}$. The latter are a direct measure of the difference in deformation potentials between the crystal ground and excited (exciton) states. The efficacy of determining such deformation potentials from the ratio of $d R / R$ to $(1 / R)$ $(\partial R / \partial \omega)_{\Omega_{u}}$ has been discussed in detail by Sell and Kane. ${ }^{23}$ From Eq. (3.7) one deduces that, if the spectral profiles of $d R / R$ and $(1 / R)(\partial R / \partial \omega)_{\Omega_{u}}$ coincide over a par- ticular photon energy range, then the exciton frequency shift $d \Omega_{u}$ is negative and corresponds to a shift of the transition to lower frequency. Noncoincidence of the former implies the existence of a strain-induced blue shift of the exciton transition. For very nearly derivative-like piezoreflection spectra one may consequently obtain a value for the magnitude of the exciton modulation sensitivity. An unambiguous absolute determination of the sign of the latter is contingent on a knowledge of both the sign of $d \Omega_{u}$, determined in the aforementioned manner, and the corresponding state of lattice strain, i. e., compression or extension. The absolute sense of the exciton strain shift may be determined with the aid of

$$
\left(\frac{d R}{R}\right)_{\mathrm{rms}}=\left(\frac{R_{\text {compression }}-R_{\text {extens ton }}}{R}\right)_{\mathrm{rms}},
$$

which may be used to amend Eq. (3.7) to

$$
\left(\frac{d R}{R}\right)_{\mathrm{rms}}=-\frac{1}{R}\left(\frac{\partial R}{\partial \omega}\right)_{\Omega_{u}} d \Omega_{\mathrm{u}_{\mathrm{rms}}},
$$

where $(d R / R)_{\text {rms }}$ represents the root mean square value of the synchronously detected output signal corresponding to a compressive-extensive deformation cycle of the crystal. Consequently, from Eqs. (3.8) and (3.9), a coincidence of the spectral profiles of $d R / R$ and $(1 / R)$ $\times(\partial R / \partial \omega)_{\Omega_{u}}$ corresponds to a strain-induced red shift of the exciton frequency resulting from lattice compression.

\section{Kramers-Kronig transform of modulated reflectivity}

The Kramers-Kronig relations may be extended to a treatment of differential reflection spectra. The following analysis derives from that developed in earlier work. 12 (d), 24

The validity of the differential Kramers-Kronig relations requires the following:

(a) The crystal dielectric function $\bar{\epsilon}=\epsilon_{r}+i \epsilon_{i}$ undergoes a uniform perturbation $\Delta \tilde{\epsilon}=\Delta \epsilon_{r}+i \Delta \epsilon_{i}$ subsequent to a strain-induced deformation.

(b) The reflecting surface represents a discontinuous interface between two different but homogeneous media and, consequently, Fresnel's equation obtains:

$$
R=\frac{(n-1)^{2}+\kappa^{2}}{(n+1)^{2}+\kappa^{2}},
$$

in which normal incidence through vacuum is assumed. $R$ is the reflectivity and $n$ and $k$ are the real and imaginary parts of the complex refractive index, respectively.

(c) There is no rotation of the $\epsilon_{i}$ and $\epsilon_{r}$ ellipsoids.

The information in the piezoreflection spectrum embodied in $\Delta \vec{\epsilon}$ may be extracted by a Kramers-Kronig transform of $\Delta R / R$. The latter is used in conjunction with the dispersion relations to calculate the straininduced phase change $\Delta \theta\left(\omega_{i}\right)$ and ultimately $\Delta \epsilon_{Y}\left(\omega_{i}\right)$ and $\Delta \epsilon_{i}\left(\omega_{i}\right)$. It should be remarked that equations such as Eq. (3.10) as well as those differential expressions to follow are most simply written in terms of the complex refractive index $\vec{n}=n+i \kappa$. Hence, $\vec{n}$ will be used in 
lieu of $\tilde{\epsilon}$ in many of the formulas to attain algebraic simplicity. The relations necessary for the calculations of $\Delta \tilde{\epsilon}$ or $\Delta \tilde{n}$ are

$$
\rho=r e^{i \theta}=\frac{n+i \kappa-1}{n+i \kappa+1},
$$

where the reflectivity $R=r^{2}=\rho \rho^{*}$. If one calculates

$$
\begin{aligned}
& d \rho=\left(\frac{\partial \rho}{\partial r}\right)_{\theta} d r+\left(\frac{\partial \rho}{\partial \theta}\right)_{r} d \theta, \\
& d \rho=\left(\frac{\partial \rho}{\partial n}\right)_{\kappa} d n+\left(\frac{\partial \rho}{\partial \kappa}\right)_{n} d \kappa,
\end{aligned}
$$

equates the two differentials, and separates terms, the following obtains:

$$
\begin{aligned}
& \Delta n\left(\omega_{i}\right)=\frac{1}{4}\left(n^{2}-\kappa^{2}-1\right) \frac{\Delta R}{R}\left(\omega_{i}\right)-n \kappa \Delta \theta\left(\omega_{i}\right), \\
& \Delta \kappa\left(\omega_{i}\right)=\frac{1}{2} n \kappa \frac{\Delta R}{R}\left(\omega_{i}\right)+\frac{1}{2}\left(n^{2}-\kappa^{2}-1\right) \Delta \theta\left(\omega_{i}\right) .
\end{aligned}
$$

In a similar manner, $\Delta \epsilon_{r}$ and $\Delta \epsilon_{i}$ may be shown to be

$$
\begin{aligned}
& \Delta \epsilon_{r}\left(\omega_{i}\right)=\frac{1}{2} \gamma \frac{\Delta R}{R}\left(\omega_{i}\right)-\delta \Delta \theta\left(\omega_{i}\right), \\
& \Delta \epsilon_{i}\left(\omega_{i}\right)=\frac{1}{2} \delta \frac{\Delta R}{R}\left(\omega_{i}\right)-\gamma \Delta \theta\left(\omega_{i}\right),
\end{aligned}
$$

where $\gamma=n\left(n^{2}-3 \kappa^{2}-1\right)$ and $\delta=\kappa\left(3 n^{2}-\kappa^{2}-1\right)$. These expressions are analogous to those for normal incidence specular reflection given by Fresnel's equation. However, in addition to $\Delta R / R\left(\omega_{i}\right)$ and $\Delta \theta\left(\omega_{i}\right)$, calculation of the above quantities also requires a knowledge of $n\left(\omega_{i}\right)$ and $\kappa\left(\omega_{i}\right)$ obtained from the unmodulated reflection spectrum. The approach using the Kramers-Kronig relations is based strictly on experimental data and the dispersion relations, and not on any a priori knowledge of, or prescription for, the dielectric function.

The strain-induced phase change $\Delta \theta\left(\omega_{i}\right)$ is an integral transform of $\Delta R / R$ and may be calculated from

$$
\Delta \theta\left(\omega_{i}\right)=P-\frac{\omega_{i}}{\pi} \int_{0}^{\infty} \frac{\frac{\Delta R}{R}(\omega)}{\omega_{i}^{2}-\omega^{2}} d \omega,
$$

where $P$ represents the Cauchy principal value of the integral. Equation (3.18) is similar in form to that of the unmodulated expression with the exception that the numerator of the integrand has changed to $d R(\omega) / R(\omega)$. Connection to the Seraphin coefficients can be made where

$$
\begin{aligned}
& \alpha=\frac{2 \gamma}{\left(\gamma^{2}+\delta^{2}\right)}, \\
& \beta=\frac{2 \delta}{\left(\gamma^{2}+\delta^{2}\right)} .
\end{aligned}
$$

The differential Kramers-Kronig transform is considerably less sensitive to the assumed or neglected variations in the $\Delta R / R$ spectrum outside of the experimental range than is the Kramers-Kronig transform of the unmodulated reflection spectrum. The relative ease of the differential transform may be attributed, in part, to partial cancellation of contributions from distant structure in the piezomodulation spectrum due to the averaging out of the positive and negative excursions in the response. ${ }^{14(2), 22}$

\section{PIEZOMODULATION SPECTROSCOPY FOR MODEL COUPLING REGIMES}

\section{A. Oriented gas}

In this limit of no coupling between transitions, the piezomodulation produces only an oscillation of the energy levels due to the effect of change of volume with concomitant change in the crystal dielectric susceptibility. The interaction is expected to be essentially that observed from high pressure spectroscopic studies. The effect of pressure on inter-and intramolecular potentials is a fundamental concern in this case.

An isotropic medium has a volume or bulk isothermal compressibility given by

$$
*=-\left(\frac{\Delta V / V}{\Delta P}\right)_{T}
$$

where $V$ and $P$ represent volume and hydrostatic pressure, respectively. For a pressure independent compressibility, the isothermal equation of state for the medium has been given by Rice and Jortner ${ }^{25}$ as

$$
V=V_{0} e^{-A P} \text {, }
$$

with the compressional work per molecule

$$
w=V_{0}\left[\frac{1}{k}\left(1-e^{-k P}\right)-P e^{-k P}\right],
$$

which reduces to $k V_{0} P^{2}$ for $\Leftrightarrow P<1$. From this, an estimate of the order of magnitude of $w$ was found to be $w$ $\leq 0.01 \mathrm{eV}$ for pressures up to $100 \mathrm{kbar}$ and $w \leq 0.1 \mathrm{eV}$ for pressures in excess of 500 kbar. Given typical bond energies on the order of volts, this analysis indicates that the major effect of pressure is a change in intermolecular distances and orientation. For piezomodulation in the elastic regime with effective pressures of less than 50 bar, it may be safely assumed that strain has a negligible effect on the free molecule Hamiltonian.

Explanation for the piezomodulation of an oriented gas crystal is the same as that for solvent shift theories based on a consideration of ground and excited state dispersion energies ${ }^{26}$ or on Onsager dielectric theory. ${ }^{27}$

It is worthwhile to consider the dispersion energy approach to the interpretation of piezomodulation spectra of oriented gas crystals. The shift equations most often used treat the ground and excited state van der Waals energy. Approximate forms for the latter may be obtained by first averaging the squared dipole-dipole interactions over all dipole orientations:

$\left\langle\left(\frac{4 \pi}{v_{0}}\right)^{2}\left(\mu_{f}^{s} \cdot \mathrm{T}_{s s^{\prime}} \cdot \mu_{s}^{s^{\prime}}\right)^{2}\right\rangle=\frac{2}{3}\left|\mu_{f}^{s}\right|^{2}\left|\mu_{z}^{s^{\circ}}\right|^{2} R_{s s^{\prime}}^{-6}$, in which \langle\rangle indicates the appropriate average. Further,

$$
\mathrm{T}_{s s^{\prime}}=\frac{v_{0}}{4 \pi} \frac{1}{R_{s s^{\prime}}^{3}}\left(1-3 \hat{\mathbf{R}}_{s s^{\prime}} \hat{\mathrm{R}}_{s s^{\prime}}\right) e^{i \mathbf{k} \cdot \mathbf{R}_{s s^{\prime}}}
$$

and

$$
\mathbf{R}_{s s^{\prime}}=\mathbf{r}_{s^{\prime}}-\mathbf{r}_{s} \text {. }
$$


Longuet-Higgins and Pople $e^{26}$ used pair interaction expressions such as this in addition to a scheme for averaging over all virtual transitions $0 \rightarrow f$ and $0 \rightarrow g$ to develop an expression for the difference in dispersion energies between ground and excited states $D=D_{u}-D_{0}$. An order of magnitude estimate of $D$ for a crystalline material may be obtained from their expression

$$
D \cong \sum_{s^{\prime} \neq s} R_{s s^{-6}}^{-6} \alpha\left(\mu_{u}^{2}+\frac{1}{\theta} \alpha E_{u}\right),
$$

where $\alpha$ is a scalar, average, low frequency molecular electronic polarizability, $\mu_{u}$ is the modulus of the $0-u$ transition moment, and $E_{u}$ is the transition energy. This lattice sum of dispersion energy differences is comprised of two types of interactions. The first term is the transition dipole-induced dipole interaction energy. The second term is the usual van der Waals energy in a form roughly equivalent to the London expression. For nonpolar systems $D$ is negative due to the attractive nature of the forces involved and the higher excited state polarizability. Both perturbation energies stabilize the excited state relative to the ground state, consequently producing a red shift of the transition energy. The stabilization of the excited state is accompanied by a decrease in the intermolecular equilibrium distance and an increase in the gradient of the excited state potential. The expression therefore predicts that weakly coupled, single particle exciton states ought to undergo a red compressional strain shift proportional to $D$.

Solvent shift expressions derived from dielectric theory are, however, more commonly used to discuss pressure shifts than is the preceeding approach. Typical of these is the Bayliss formula ${ }^{28}$

$$
-\Delta \nu_{u}=K \frac{f_{u}}{\nu_{u} a^{3}}\left(\frac{n^{2}-1}{2 n^{2}+1}\right),
$$

where $-\Delta \nu_{u}$ indicates a red shift for a transition of frequency $\nu_{u}$ and oscillator strength $f_{u} . K$ is a collection of constants, $a^{3}$ is a molecular cavity volume, and $n$ is the refractive index at $\nu_{u}$. Isotropic pressure shift data may be fit by the Bayliss formula if the bulk compressibility of the medium is known. The ClausiusMossotti equation is used to ascertain the density dependence of $\tilde{\epsilon}$.

Thus, the piezomodulation spectrum in this limiting case is expected to reduce simply to the logarithmic derivative [Eq. (3.7)] of the unmodulated reflection spectrum. The only advantage gained here from piezomodula tion spectroscopy is a less noisy derivative spectrum than is usually obtained by calculation from the direct spectrum. The result is equivalent to external wavelength modulation. Of course, any deviation would be indicative of some resonance force coupling in the crystal.

\section{B. Weak to intermediate coupling}

In weak to intermediate coupling systems, both the analytic dipole interactions and the van der Waals shifts must be considered in detail. The problem is essential ly that of the variance of the dispersion energy and reso- nance interaction with variation of lattice constants. This has been treated in effect by Davydov in his discussion of the coupling between excitons and the lattice vibrations. ${ }^{29}$ The dependence of the matrix elements of these interactions upon displacements of the molecules from their equilibrium positions was expressed in the Hamiltonian:

$$
\mathcal{H}=\mathfrak{H}_{\text {ex }}(0)+W(R)+\mathcal{H}^{(1)}+\mathcal{H}^{(2)} \text {, }
$$

where the operator for the undeformed lattice is

$$
\mathcal{H}_{e x}(0)=\sum_{n}[\Delta e+D(0)] B_{n}^{+} B_{n}+\sum_{n, m}^{\prime} M_{n m}(0) B_{n}^{+} B_{m},
$$

where

$$
D(0)=D_{u}(0)-D_{0}(0)
$$

Here $B_{n}^{+}$and $B_{n}$ are the exciton creation and annihilation operators, respectively, and $D(0)$ gives the change in the interaction energy of the molecules in the undistorted crystal with a molecule in its excited state

$$
D(0)=\sum_{m \neq n}\left(\left\langle 0 u\left|V_{n m}\right| u 0\right\rangle-\left\langle 00\left|V_{n m}\right| 00\right\rangle\right),
$$

with $V_{n m}$ the Coulomb interaction operator. Also, $\Delta e$ is the excitation energy of an isolated molecule. The interaction leading to transfer of excitation is given by

$$
M_{n m}=\left\langle 0 u\left|V_{n m}\right| 0 u\right\rangle \text {. }
$$

$W(R)$ determines the potential energy of interaction of molecules in a lattice devoid of excitons:

$$
W(R)=W(0)+\frac{1}{2} \sum U_{n m}^{i j} R_{n}^{i} R_{m}^{j},
$$

with $U$ the potential and the $R$ 's the deviations from equilibrium positions. The $n$ and $m$ label the molecular positions and the $i$ and $j$ designate the three translations and three rotations of displacement in an adiabatic approximation. The two perturbation terms $\mathcal{H}^{(1)}$ and $\mathrm{JC}^{(2)}$ describe the exciton-lattice (phonon) interaction. $\mathfrak{H}^{(1)}$ describes the scattering of a constant number of excitons by a changing number of phonons:

$\mathcal{H}^{(1)}=\sum_{n, m}^{\prime} B_{n}^{+} B_{m} \sum_{j=1}^{6}\left[\left(R_{n}^{j} \frac{\partial}{\partial R_{n}^{j}}+R_{m}^{j} \frac{\partial}{\partial R_{m}^{j}}\right) M_{n m}\right]_{0}$,

with $\mathfrak{H}^{(2)}$ giving the displacement from the equilibrium positions of the molecules over the region of the excitation

$\mathfrak{H}^{(2)}=\sum_{n, m}{ }^{\prime} B_{n}^{+} B_{n} \sum_{j=1}^{6}\left[\left(R_{0}^{j} \frac{\theta}{\partial R_{0}^{j}}+R_{m}^{j} \frac{\theta}{\partial R_{m}^{j}}\right) D_{0 m}(R)\right]_{0}$.

However, this formalism was not developed with a view toward strain effects on excitons.

Schipper ${ }^{30}$ has developed an exciton-strain coupling theory which may be regarded as a development of the approach of Davydov and which is relevant to weak and intermediate coupling strength systems. Attention was specifically directed to the influence of static strain on the lowest singlet vibronic exciton spectra of an- 
thracene and naphthalene.

The vibronic exciton factor group state energies derived from the $0,0 \rightarrow u, r$ molecular vibronic transitions are written as

$$
E_{ \pm}^{u_{u} r}=E_{ \pm}^{1 u_{,} r}+E_{ \pm}^{2 u_{,} r},
$$

where the 1 or 2 superscript label denotes the order of the perturbation energy and

$$
\begin{aligned}
& E_{ \pm}^{1 u_{r} r}=\Delta e_{u_{r} r}+D+\xi_{u_{,} r}^{2}\left(i_{\alpha \alpha} \pm i_{\alpha \beta}\right), \\
& E_{ \pm}^{2 u_{\mu} r}=\sum_{t \neq \mu} \frac{\xi_{u_{1} r}^{2}\left(i_{\alpha \alpha}^{u_{\alpha} t} \pm i_{\alpha \beta}^{u_{0} t}\right)^{2}}{\left(E_{ \pm}^{1 u_{t} r}-E_{ \pm}^{1 t}\right)},
\end{aligned}
$$

in which $\xi_{u_{r}}^{2}$ is the relevant Franck-Condon factor and the $i_{\alpha \alpha}$ and $i_{\alpha \beta}$ are the analytic interaction energies between equivalent and inequivalent sites, respectively. The plus sign designates a factor group state containing the macroscopic dipole interaction (nonanalytic part of the lattice sum ${ }^{31}$ ) whereas the minus state is devoid of the interaction. The second order perturbation expression [Eq. (4.18)] spans all excited states $t \neq u$ but neglects the vibrational structure of the latter. The perturbation development is restricted to a consideration of the two lowest energy singlet exciton states. The straininduced shift of $E^{u_{r} r}$ may be written in terms of dif ferential changes in the van der Waals and exciton resonance interactions as

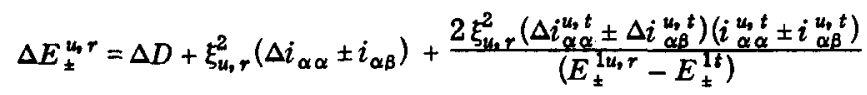

$$
\begin{aligned}
& -\frac{\xi_{u_{\alpha} r}^{2}\left[\left(\Delta i_{\alpha \alpha}^{u \mu} \pm \Delta i_{\alpha \beta}^{u u \mu}\right)-\left(\Delta i_{\alpha \alpha}^{t t} \pm \Delta i_{\alpha \beta}^{t t}\right)\right]\left(i_{\alpha \alpha}^{u_{0} t} \pm i_{\alpha \beta}^{u_{s} t}\right)^{2}}{\left(E_{ \pm}^{1 u_{,} r}-E_{ \pm}^{1 t}\right)^{2}},
\end{aligned}
$$

in which the strain-induced changes in the macroscopic dipole interactions are not explicitly considered. One may write the strain-induced vibronic exciton shifts in differential form as

$$
d E_{ \pm}^{u_{1} r}=\sum_{i=1}^{6} E_{ \pm}^{\prime}\left(u r, v_{i}\right) d v_{i},
$$

where

$$
E_{ \pm}^{\prime}\left(u r, v_{i}\right)=\left(\frac{\partial E_{ \pm}^{u_{i} r}}{\partial v_{i}}\right)
$$

and the $v_{i}$ represent the six unit cell parameters. The exciton energy derivatives $E_{ \pm}^{\prime}\left(u r, v_{i}\right)$ are expanded in terms of intermolecular distance and orientation derivatives.

The differential modulation sensitivity of the vibronic exciton states which derives from the differences in Franck-Condon factors $\xi_{\mu_{2}}^{2}$ among the latter is apparent from expression (4.19). The differences in the vibronic exciton strain shifts are, however, small and obscured by the rather large van der Waals differential shifts $d D$ for the particular case of anthracene. The reported theoretical energy strain derivatives may then be used to calculate the exciton shifts from

$$
d E_{ \pm}^{u_{t} r}=E_{ \pm}^{\prime}(u r, a) d a+E_{ \pm}^{\prime}(u r, b) d b ;
$$

which considers strains in the $a b$ crystal plane and disregards shearing. Equation (4.22) requires a knowledge of $d a$ and $d b$ which may be ascertained for the strain configuration employed in the piezoreflection experiments.

The differential shift $d E^{\mu_{\text {r }}}$ is written in terms of contributions from $d D$ and $d i$ which may be calculated by performing the appropriate derivative lattice sums. The pairwise interactions are also assumed to be capable of expansion in a Taylor series about the unstrained lattice parameters in which only the linear terms are considered.

The crystal group state dispersion energy per molecule is represented as

$$
D_{0}=-\sum_{s^{\prime} \neq s} \frac{1}{2} \sum_{f, s} \frac{\frac{2}{3}\left|\mu_{f}^{s}\right|^{2}\left|\mu_{f}^{s^{\prime}}\right|^{2} R_{s s^{\prime}}^{-6}}{\hbar\left(\omega_{f}+\omega_{g}\right)},
$$

where the numerator represents the result of spherical ly averaging the pairwise, squared transition dipole interaction. The summation over all transitions $0 \rightarrow f$ and $0 \rightarrow g$ is approximated by the familiar London formula and the result is then written for a pair interaction as

$$
-\frac{3}{4} I \alpha_{0}^{2} R_{s s^{\prime}}^{-6}=A^{0} R_{s s^{\circ}}^{-6},
$$

where $I$ represents the first ionization potential and $\alpha_{0}$ is the static polarizability. An analogous formula obtains for the $u$ th excited state, leading to the difference in dispersion energies between states 0 and $u$ expressed as

$$
D=D_{u}-D_{0}=\sum_{s^{\prime} \neq s} \frac{A^{0 u}}{R_{s s^{\prime}}^{6}}=\sum_{s^{\prime} \neq s} D_{s s^{\prime}},
$$

where

$$
A^{0 u}=A^{u}-A^{0} \text {. }
$$

The parameter $A^{0 u}$ is defined by calculating the appropriate $R_{s s}^{-6}$, lattice sum and equating the result to $D$ which is assigned a value equal to the vapor-to-crystal transition energy shift excluding that contribution to the latter which is due to exciton resonance. The strain differential, which is also parametrized in terms of the empirically determined $A^{0 u}$, is expressed (for two molecules in the unit cell) as

$$
\begin{aligned}
d D & =\sum_{s^{\prime} \neq s} d D_{s s^{\prime}} \\
& =\sum_{s^{\prime} \neq s}\left[\sum_{i}^{6} D_{s s^{\prime}}^{\prime}\left(v_{i}\right) d v_{i}+\sum_{j}^{3} D_{s s^{\prime}}^{\prime}\left(x_{j}^{s s^{\prime}}\right) d x_{j}^{s s^{\prime}}\right],
\end{aligned}
$$

where $v_{i}$ labels the $i$ th unit cell parameter and the $x_{j}^{s s^{\circ}}$ $=x_{j}^{s^{\prime}}-x_{j}^{s}$ are the displacements. The latter quantity specifically pertains to those changes in intermolecular distance due to internal (to the unit cell) relaxation subsequent to a change in unit cell size or shape, i.e., a change in $v_{i}$. Furthermore, expression (4.26) neglects any change in $D$ due to a strain-induced molecular reorientation. The dispersion derivatives $D_{s s^{\prime}}^{\prime}\left(v_{i}\right)$ have the form

$$
D_{s s^{\circ}}^{\prime}\left(v_{i}\right)=\left(\frac{\partial D_{s s^{\prime}}}{\partial v_{i}}\right)=\left(\frac{-6 A^{0 u}}{R_{s s^{\prime}}^{7}}\right)\left(\frac{\partial R_{s s^{\prime}}}{\partial v_{i}}\right) .
$$

The evaluation of the corresponding differential change

$$
d D_{s s^{\prime}}\left(v_{i}\right)=D_{s s^{\prime}}^{\prime}\left(v_{i}\right) d v_{i}
$$


requires a knowledge of $d v_{i}$. This quantity must, in general, be ascertained from a knowledge of the elastic constant tensor of the crystal and the externally applied stress. The equations which facilitate the calculation of of crystal strains have been given by Schipper.

Therefore, neglecting the orientational derivatives, the differential change in a given pair interaction $d D_{s s^{\prime}}$ requires the evaluation of nine differentials. The total change $d D$ may be subsequently obtained by performing the lattice sum of Eq. (4.26). If one considers only changes in the lattice parameters $a, b$, and $c$, that expression becomes

$d D=\sum_{s^{\prime} \neq s^{s}}\left[D_{s s^{\prime}}^{\prime}(a) d a+D_{s s^{\prime}}^{\prime}(b) d b+D_{s s^{\prime}}^{\prime}(c) d c\right]$

$d D=D^{\prime}(a) d a+D^{\prime}(b) d b+D^{\prime}(c) d c$.

Since the unit cell strains such as $d a / a$ may be deduced for the strain configuration employed in piezomodulation experiments, it is possible to calculate $d D$ due to straininduced unit cell changes semiempirically. The latter may then be compared to the experimentally determined exciton frequency shifts which may be obtained from a comparison of $\Delta R / R$ to the logarithmic frequency derivative of $R$.

The expressions for the differential changes in the analytic dipole interaction energy are similar in form to those used to evaluate $d D$. The neglect of the orientational strain derivatives which facilitates the calculation is, in general, not a sound assumption insofar as the analytic dipole interactions are concerned. Consequently, for crystals with two molecules per unit cell (such as anthracene), there are 15 differential changes which must be considered in the evaluation of $d i_{s s^{\prime}}$. The pertinent expressions for the latter may be given considering only degenerate level coupling. The more general formulas may be obtained in the original paper.

The dipole interaction energy may be written as

$$
i_{s s^{\prime}}=\frac{\mu^{2}}{R_{s s^{\prime}}^{3}} F_{s s^{\prime}}=\mu^{2} G_{s s^{*}},
$$

where $F_{s s}$, is the appropriate combination of direction cosines. The differential change in $i_{s s^{\prime}}$ may be written as

$$
d i_{s s^{\prime}}=\mu^{2} d G_{s s^{\prime}} .
$$

The unit cell parameter derivatives $G_{s s}^{\prime} \cdot\left(v_{i}\right)$ have the form

$$
G_{s s^{\prime}}^{\prime}\left(v_{i}\right)=R_{s s^{\prime}}^{-3}\left[\left(\frac{\partial F_{s s^{\prime}}}{\partial v_{i}}\right)-\frac{3 F_{s s^{\prime}}}{R_{s s^{\prime}}}\left(\frac{\partial R_{s s^{\prime}}}{\partial v_{i}}\right)\right]
$$

and the derivatives with respect to orientation coordinates are defined analogously. Consequently, the evaluation of the differential changes in the analytic dipole interaction energies involves lattice sums of the type

$$
\sum_{s^{\prime} \neq s} G_{s s^{\prime}}^{\prime} e^{\boldsymbol{i k \cdot \mathbf { R } _ { s s ^ { \prime } }},}
$$

which have been calculated by Schipper for anthracene and naphthalene using planewise summation techniques.
This development of the exciton-strain coupling gives a framework sufficient to develop an analysis of modulated piezoreflection of molecular crystals in the weak to intermediate coupling regime. Although the theoretical concepts outlined in this section lead to an acceptable interpretation of piezospectroscopic results, the latter may be expected to lead to further development of the theory of strain effects on molecular exciton states.

\section{Strong coupling}

Large dipole strength molecular transitions ( 2-4 $\AA^{2}$ ) are the progenitors of strongly coupled crystal eigenmodes. Thus, a more detailed consideration of the dielectric function is required. An investigation of the lattice strain dependence of the exciton parameters which appear in $\tilde{\epsilon}$ is necessary to the discussion of the optical data. The classical dipole formulation of exciton states developed by Mahan ${ }^{32}$ and extended by Philpott ${ }^{31}$ provides a many order perturbation description of the coupled crystal excitations which is conceptually straightforward and distinctively physical.

The expressions for the calculation of the vibronic exciton frequencies in the classical dipole formalism are necessary to the analysis of piezoreflection in this regime. For the case in which the transition dipole $\hat{\mu}$, the polarization $\hat{e}$, and wave vector of the light $\hat{k}$ are coplanar, the Coulomb exciton frequencies are determined from ${ }^{31}$

$1+\sum_{u} \frac{2 \omega_{u} \xi_{u}^{2}}{\hbar\left(\omega_{u}^{2}-\omega^{2}+i \gamma_{u} \omega\right)}\left[i(0)+\frac{4 \pi \mu^{2}}{\epsilon_{0} v_{0}}(\hat{\mu} \cdot \hat{\mathbf{k}})^{2}\right]=0$

and the longitudinal Coulomb exciton frequencies $(k=0$ polaritons) are the roots of

$$
1+\sum_{u} \frac{2 \omega_{u} \xi_{u}^{2}}{\hbar\left(\omega_{u}^{2}-\omega^{2}+i \gamma_{u} \omega\right)}\left[i(0)+\frac{4 \pi \mu^{2}}{\epsilon_{0} v_{0}}\right]=0 .
$$

$i(0)$ represents the analytic part of the dipole coupling energy at $\mathbf{k}=0$ and the macroscopic interactions are screened by a background dielectric constant $\epsilon_{0,}{ }^{31}$

The phenomenological consequences of excitonphoton coupling for a stack of vibronic polaritons are shown schematically in Fig. 2 for weak, intermediate, and strong coupling. For weak coupling, the polariton stopping band is narrow and less than the FranckCondon bandwidth of the corresponding molecular transition. In the strong coupling regime, the very wide stop band is typically an order of magnitude greater than the free molecule vibrational spacing. The reflection spectrum observed for the (010) face of TCNQ, for example, indicates a crystal bandwidth of approximately $14000 \mathrm{~cm}^{-1}$ whereas the vibronic level spacing is $\approx 1400 \mathrm{~cm}^{-1}$. ${ }^{33}$ The width of the Coulomb exciton band depends on the plasma frequency squared $\omega_{0}^{2}$. For large values of the latter the crystal excitation bandwidth exceeds the natural or Franck-Condon bandwidth and, consequently, becomes a parameter which must be considered when describing the strain modulation of the dielectric function. The simple one level system suf fices to describe the important features of polariton bandwidth modulation. 

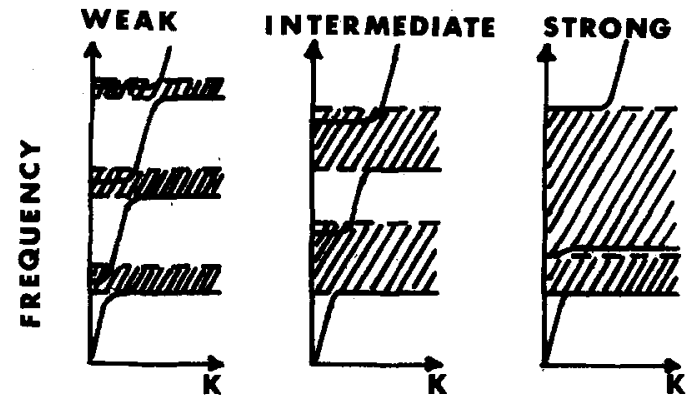

FIG. 2. Polariton dispersion relations for several vibronic transitions and different coupling strengths [after Philpott (Ref. 31)].

\section{One level system}

For very strong coupling, the lattice polarization wave may be characterized as purely electronic in nature and all the transition intensity is concentrated in a single resonance. The crystal oscillator strength $F_{u}$ equals the molecular electronic transition oscillator strength $f$. One may readily calculate the exciton frequencies from Eqs. (4.35) and (4.36):

$$
\begin{aligned}
& \Omega^{2}=\omega_{u}^{2}+\frac{2 \omega_{u}}{\hbar}\left[i(0)+\frac{4 \pi \mu^{2}}{\epsilon_{0} v_{0}}(\hat{\mu} \cdot \hat{\mathbf{k}})^{2}\right], \\
& \Omega_{L}^{2}=\omega_{u}^{2}+\frac{2 \omega_{u}}{\hbar}\left[i(0)+\frac{4 \pi \mu^{2}}{\epsilon_{0} v_{0}}\right],
\end{aligned}
$$

where $\Omega_{L}^{2}$ is the longitudinal exciton frequency and $\Omega^{2}$ is the Coulomb exciton frequency $(\epsilon \rightarrow \infty)$ which depends on the direction of $\hat{\mathbf{k}}$. For a transverse exciton $\hat{\mu} \cdot \hat{\mathbf{k}}$ $=0$, one may write

$$
\Omega_{T}^{2}=\omega_{u}^{2}+\frac{2 \omega_{u}}{\hbar} i(0)
$$

Consequently, the crystal bandwidth is

$$
\hbar\left(\Omega_{L}-\Omega_{T}\right)=w \simeq \frac{4 \pi \mu^{2}}{\epsilon_{0} v_{0}} .
$$

Figure 3 illustrates the dispersion of the polariton frequency with $\mathbf{k}$ for a one level system and depicts the relationship between the latter and the transverse and longitudinal Coulomb exciton frequencies. The strain modulation of the stopband width may be simply written as

$$
\begin{aligned}
& d w=-\frac{4 \pi \mu^{2}}{\epsilon_{0} v_{0}} \frac{d v_{0}}{v_{0}}, \\
& d w=-w \frac{d v_{0}}{v_{0}},
\end{aligned}
$$

which is a genuinely macroscopic energy modulation depending only on $v_{0}$ and not the microscopic structure of the crystal.

A useful model system for an anisotropic molecular crystal is an infinite cubic crystal comprised of anisotropic molecules. For such a system, the dipole lattice sum becomes

$$
T(\mathbf{k})=(\hat{\mu} \cdot \hat{k})^{2}-\frac{1}{3} \text {. }
$$

Therefore, the transverse and longitudinal exciton en- ergies for a one level system may be written as

$$
\begin{aligned}
& E_{r} \simeq \Delta e_{u}+D-\frac{4 \pi \mu^{2}}{3 \epsilon_{0} v_{0}}, \\
& E_{L} \simeq \Delta e_{u}+D+\frac{8 \pi \mu^{2}}{3 \epsilon_{0} v_{0}},
\end{aligned}
$$

in which the van der Waals shift term $D$ is now made explicit and $\Delta e_{u}$ is the free molecule transition energy. From this expression one deduces that the piezomodulation results in both a stopping band modulation and a mean shift of the entire Coulomb exciton band. Since the transverse-longitudinal splitting is asymmetric about the site-shifted molecular excitation energy, the strain-induced shift of the mean polariton energy has contributions from both $d D$ and an additional term proportional to $d v_{0} / v_{0}$. The differential exciton energy shift expressions for the one level, cubic crystal model system are then

$$
\begin{aligned}
& d E_{T}=d D+\frac{4 \pi \mu^{2}}{3 \epsilon_{0} v_{0}} \frac{d v_{0}}{v_{0}}, \\
& d E_{L}=d D-\frac{8 \pi \mu^{2}}{\epsilon_{0} v_{0}} \frac{d v_{0}}{v_{0}}, \\
& d w=-\frac{4 \pi \mu^{2}}{\epsilon_{0} v_{0}} \frac{d v_{0}}{v_{0}}, \\
& d E_{\text {mean }}=d D-\frac{2 \pi \mu^{2}}{3 \epsilon_{0} v_{0}} \frac{d v_{0}}{v_{0}} .
\end{aligned}
$$

\section{Two vibronic level system}

The general equations from the classical dipole theory of excitons facilitate the numerical calculation of $\Omega_{u}^{2}$, $F_{u}$, and $\tilde{\epsilon}(\omega, \mathbf{k})$ for any degree of coupling in a $n$-vibronic level system. Analytic expressions for the evaluation of exciton parameters may be obtained, however, only for the one and two vibronic exciton state systems. The physical insights into exciton modulation mechanisms to be realized from the solutions of the latter problem are valuable in shaping a more detailed analysis. It is useful to obtain closed-form expressions for the exciton parameters $\tilde{\epsilon}(\omega, k)$ and their corresponding differentials for the two vibronic level system.

The differential quantities obtained in the Appendix may be used to calculate a model piezoreflection spectrum comprised of two vibronic exciton resonances.

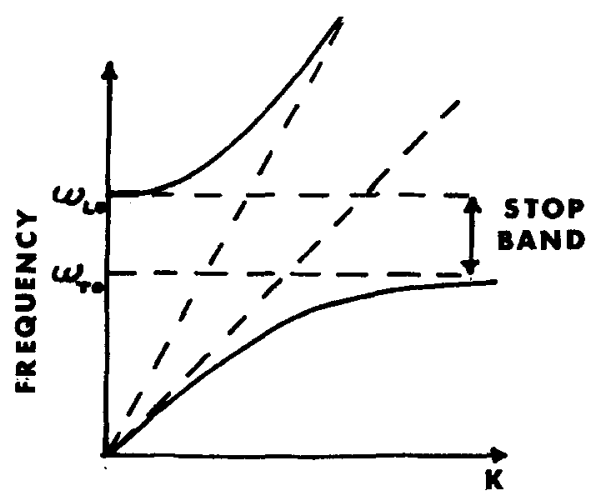

FIG. 3. Polariton dispersion for the one level system. 
The recognition of contributions from the latter to the modulation of the dielectric function is facilitated by the factorization of $d \vec{\epsilon}$ into the following differential quantities:

$$
\begin{aligned}
& d \bar{\epsilon}=\left(\frac{\partial \bar{\epsilon}}{\partial \Omega_{u}^{2}}\right)_{F_{u^{0}} \omega_{0}^{2}, \epsilon_{0}} d \Omega_{u}^{2}+\left(\frac{\partial \bar{\epsilon}}{\partial F_{u}}\right)_{\Omega_{u^{2}, \omega_{0}^{2}, \epsilon_{0}}^{2}} d F_{u} \\
& +\left(\frac{\partial \bar{\epsilon}}{\partial \omega_{0}^{2}}\right)_{\Omega_{u^{\prime}}^{2}, F_{u^{\prime}} \epsilon_{0}} d \omega_{0}^{2}+\left(\frac{\partial \tilde{\epsilon}}{\partial \epsilon_{0}}\right)_{\Omega_{u^{2}}^{2}, F_{u}, \omega_{0}^{2}} d \epsilon_{0}=\sum_{P=1}^{4}\left(\frac{\partial \bar{\epsilon}}{\partial P}\right) d P,
\end{aligned}
$$

where

$$
\left(\frac{\partial \epsilon_{i}}{\partial \epsilon_{0}}\right)=0 \text { and } P=\Omega_{u}^{2}, F_{u}, \omega_{0}^{2}, \epsilon_{0} .
$$

For simplicity, the strain modulation of $\gamma_{u}$ and $(\hat{\mu} \cdot \hat{e})^{2}$ has been neglected. The expression for $d \Omega_{ \pm}^{2}[\mathrm{Eq}$. (A8)] contains no explicit modulation of the dipole lattice sum $T(\mathbf{k})$. Such a modulation is, however, present by virtue of the $d v_{0} / v_{0}$ term. $T(\mathbf{k})$ has been approximated as in the one level model [Eq. (4.42)]. The analytic part of the lattice sum, in contrast to the cubic crystal case, does not vanish by symmetry for anisotropic molecular crystals. Equation (A8) expresses the strain-induced exciton shifts in terms of a modulation of macroscopic dipole interactions which depend only on $v_{0}$ and dif ferential changes in the van der Waals shifted molecular excitation frequencies. The exciton resonance shift formula is also based on the assumption that the molecular oscillator strengths $f_{1}$ and $f_{2}$ are insensitive to small changes in intermolecular distance and orientation. Implicit in this assumption is the neglect of strain effects on both the intramolecular potential and the molecular excitation frequencies $\omega_{1}$ and $\omega_{2}$ which are present in the oscillator strength expressions. Since $\omega_{1}$ and $\omega_{2}$ are site-shifted excitation frequencies, it is not entirely permissible to neglect that part of the molecular oscillator strength change which arises from the strain perturbation of $\omega_{1}$ and $\omega_{2}$. Contributions from the latter to $d f_{1}$ or $d f_{2}$ are, to a first approximation, reasonably assumed to be negligible. The strain modulation of the site-shifted molecular excitation frequencies $d \omega_{1}$ and $d \omega_{2}$ may be calculated semiempirically from a knowledge of the vapor-to-crystal transition energy shift which may be used to parametrize the dispersion derivative lattice sums.

The expressions for $\Omega_{ \pm}^{2}$ and $F_{ \pm}$and their corresponding differentials were used in calculation of the modulated piezoreflection spectrum of a typical strongly coupled system:

$\frac{d R}{R}=\sum_{P=1}^{4} \frac{1}{R}\left(\frac{\partial R}{\partial P}\right) d P=\sum_{P=1}^{4}\left[\alpha\left(\frac{\partial \epsilon_{r}}{\partial P}\right) d P+\beta\left(\frac{\partial \epsilon_{i}}{\partial P}\right) d P\right]$.

This permits analysis of the modulation spectrum in terms of contributions of the various dielectric theory parameters. The results are displayed in Fig. 4. Care should be taken by recognizing that the ordinate scales vary by more than an order of magnitude. The calculated reflection spectrum for the model system is also shown. The top spectrum is that of the total function. The calculated spectra indicate the occurrence of

\section{NANOMETERS}

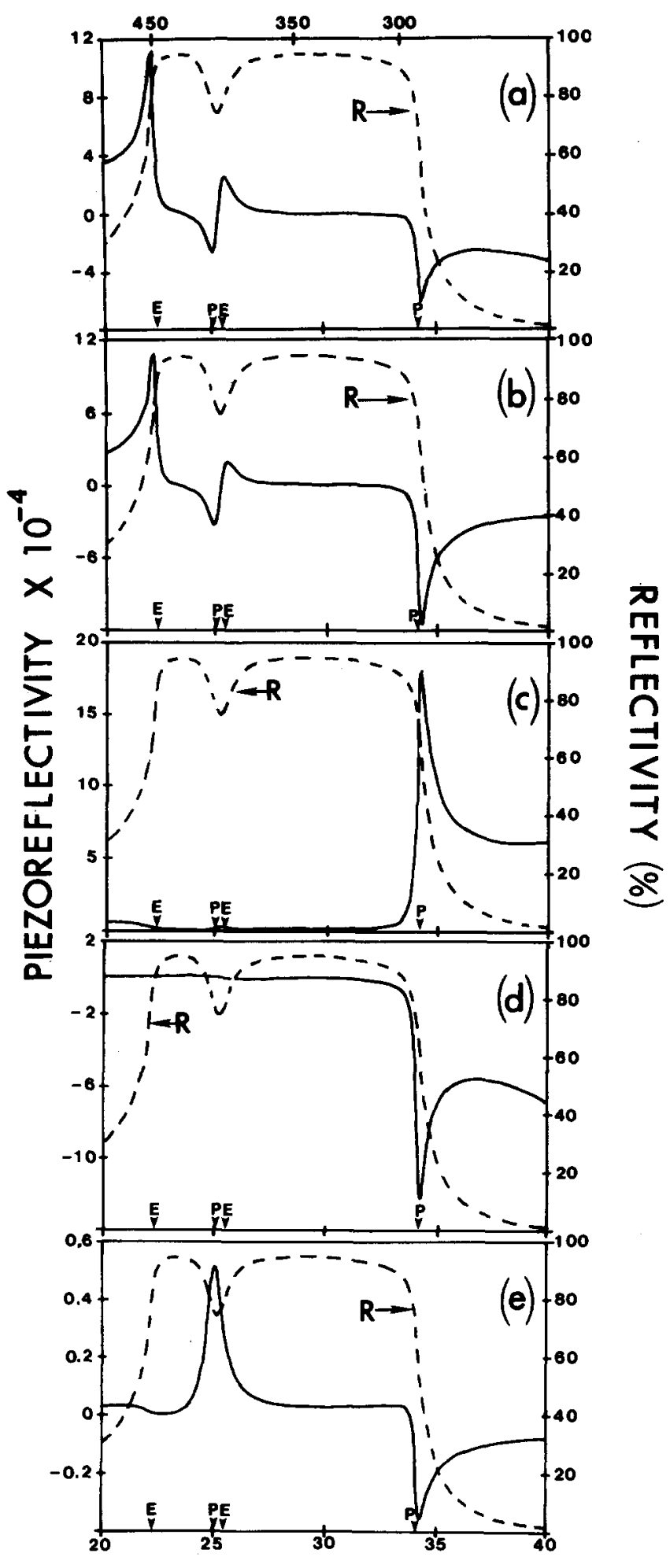

\section{KILOKAYSERS}

FIG. 4. Calculated piezoreflection spectra for a strongly coupled, two vibronic exciton system with the photon wave vector transverse to the transition moment. Figure 4 (a) represents the composite $\Delta R / R$ spectrum (solid line) of the model reflection band (dashed line). Figures $4(\mathrm{~b}), 4(\mathrm{c}), 4(\mathrm{~d})$, and $4(\mathrm{e})$ correspond to $(\Delta R / R)_{P}=(1 / R)(\partial R / \partial P) \Delta P$, where the modulation parameter $P$ is $\Omega_{u}^{2}, \omega_{0}^{2}, \epsilon_{0}$, and $F_{u}$, respectively. $E$ and $P$ locate the positions of the transverse and longitudinal (or $k=0$ polariton) excitons, respectively. 
a red shift under compression. As expected, the modulation of the exciton oscillator strength is of least conse quence. The strain shift of the exciton frequency is the dominant interaction over most of the bandwidth. The modulation effects at the Drude type edge are of interest since here the strongest response is observed in the behavior of all of the parameters. It may be expected that deviations from model behavior may then be most detectable at this edge. Thus, the general band shape of the piezomodulation spectrum in the strong coupling case has been obtained. This shape, however, will be strongly dependent upon that of the reflection spectrum.

Of particular interest is the behavior of the piezomodulation at the $\mathbf{k}=0$ polaritons. In reflection spectra these modes occur at critical points in the joint density of states and thus extrema in the piezomodulation spectra are expected to be associated with these modes. ${ }^{14(a), 34}$ Since the frequency associated with the $k=0$ polariton is constant (for small spatial dispersion), the observation of extrema in the piezoreflection spectra which are located at the same frequency in the various axial spectra would be expected to locate $\mathbf{k}=0$ polaritons.

It is straightforward to apply the formalism to obtain the response for other coupling regimes from the same formalism, but in those cases the spectrum is mainly derivative like and dominated by the $d \Omega_{u}$ term. Further, the microscopic approach is also more physically revealing.

\section{CONCLUSIONS}

A method for the measurement and interpretation of the effect of strain on the spectra of molecular crystals in the limits of strong, weak, and vanishingly small coupling has been presented. The model has been a straightforward one which applies classical dipole interaction theory to the modulation of the exciton states. The physical picture is one of the variance of the dispersion energy and exciton resonance interaction due to the modulation of the dipole-dipole interactions by the oscillatory displacement of the molecules from their equilibrium orientations. The dispersion or, equi valently, volume modulation is common to all coupling regimes and is, in fact, the only one in the limit of the oriented gas. In the intermediate and weak coupling regimes, the modulated lattice sum becomes of importance and may be so in the case of strong coupling if the analytic lattice sum is comparable to the nonanalytic term.

The piezomodulation of reflection spectra can be used to obtain directly the deformation potentials in the elastic limit for vibronic states. A microscopic theory exists to which the measured values may be compared. The technique is also useful for enhancement of structure. ${ }^{35}$

Because of the low modulation frequencies, the per turbation is homogeneous. The experiment may be designed to give uniaxial stress or a strain field which is isotropic in a plane of the crystal; other configurations are possible. The vectorial property of the perturbation makes it an effective tool for probing de- generate states since the strain can be applied in specific crystal directions to yield lower symmetry, e.g., along [111] in a cubic crystal. Thus, piezomodulation spectroscopy has obvious application to the study of the spectra of single crystals of inorganics where the need of doping the ion of interest into a host lattice with all of the attendant uncertainties is obviated. Further, it will permit more detailed study of crystal interactions in the neat crystal.

A further ramification of the technique is the modulation of luminescence which has already been demonstrated. ${ }^{36}$ Because the strain transducers can be driven to the $\mathrm{MHz}$ range, it would be expected that processes which occur on the time scale of the period of the applied perturbation, say triplet exciton fusion, would interact strongly with the perturbation and that this would depend upon whether the modulation was on a time scale less than, greater than, or equal to the lifetime of the excitonic process. The method may also be expected to afford more quantitative study of triboluminescent crystals. ${ }^{37}$

The directional property of the modulation can also be used for the study of the effect of defects on the optical or photochemical properties. For example, crystals which react at defects should show enhanced strain sensitivity at these sites and thus an enhanced piezooptical signal. ${ }^{38}$ The application to the spectroscopic study of other defects, color centers, etc. is obvious.

Thus, piezomodulation spectroscopy has great promise in application to many problems in solid state chemistry. While the discussion of its range of applicability is far from exhaustive, its versatility is demonstrated.

\section{ACKNOWLEDGMENTS}

This research was supported by the National Science Foundation through Grants NSF DMR-79-08-759, and by the Research Council of the University of Nebraska.

\section{APPENDIX: MODULATION VARIABLES OF THE DIELECTRIC FUNCTION}

The two vibronic exciton roots $\Omega_{ \pm}^{2}$ and associated oscillator strengths $F_{ \pm}$are labeled by \pm , which also designates the arithmetic operation to be performed in the algebraic expression used to evaluate the corresponding parameter. The 1,2 designation of distinctly molecular quantities labels the $(0,0)$ and $(0,1)$ vibronic transition parameters. Carets designate unit vectors. Using the basic equations of the classical dipole formulation, ${ }^{31}$ the two vibronic exciton frequencies for the two level system are given by

$$
\Omega_{ \pm}^{2}=\frac{1}{2}\left\{\left(\omega_{1}^{2}+\omega_{2}^{2}\right)+\left[\omega_{0}^{2} f_{1} \mathbf{T}(\mathbf{k})+\omega_{0}^{2} f_{2} \mathbf{T}(\mathbf{k})\right] \pm \Delta\right\},
$$

where $\Delta$ is defined by

$$
\begin{aligned}
\Delta= & \left\{\left(\omega_{1}^{2}-\omega_{2}^{2}\right)^{2}+2\left(\omega_{2}^{2}-\omega_{1}^{2}\right)\left[\omega_{0}^{2} f_{2} \mathbf{T}(\mathbf{k})-\omega_{0}^{2} f_{1} \mathbf{T}(\mathbf{k})\right]\right. \\
& \left.+\left[\omega_{0}^{2} f_{1} \mathbf{T}(\mathbf{k})+\omega_{0}^{2} f_{2} \mathbf{T}(\mathbf{k})\right]^{2}\right\}^{1 / 2},
\end{aligned}
$$

where the lattice sum $\mathbf{T}(\mathbf{k})$ may be taken in its most general form ${ }^{31}$ and $\omega_{0}^{2}=z 4 \pi e^{2} / m v_{0} \epsilon_{0}$, where $z$ represents the number of molecules in the unit cell of volume $v_{0}$. The corresponding oscillator strengths are 


$$
F_{ \pm}=\frac{\left(\Omega_{ \pm}^{2}-\omega_{1}^{2}\right)^{2}\left(\Omega_{ \pm}^{2}-\omega_{2}^{2}\right)^{2}}{\left(\Omega_{ \pm}^{2}-\omega_{2}^{2}\right)^{2} \omega_{0}^{4} T^{2}(\mathbf{k}) f_{1}+\left(\Omega_{ \pm}^{2}-\omega_{1}^{2}\right)^{2} \omega_{0}^{4} T^{2}(\mathbf{k}) f_{2}} .
$$

Consequently, a judicious selection of $\epsilon_{0}$ and $\gamma_{ \pm}$allows the crystal dielectric function to be calculated from

$$
\epsilon_{r}=\epsilon_{0}\left[1+\frac{\omega_{0}^{2} F_{+} P \Omega_{1}}{\left(\Omega_{1}^{2}+\gamma_{+}^{2} \omega^{2}\right)}+\frac{\omega_{0}^{2} F_{-} P \Omega_{2}}{\left(\Omega_{2}^{2}+\gamma_{-}^{2} \omega^{2}\right)}\right]
$$

and

$$
\epsilon_{i}=\epsilon_{0}\left[\frac{\gamma_{+} \omega \omega_{0}^{2} F_{+} P}{\left(\Omega_{1}^{2}+\gamma_{+}^{2} \omega^{2}\right)}+\frac{\gamma_{-} \omega \omega_{0}^{2} F_{-} P}{\left(\Omega_{2}^{2}+\gamma_{-}^{2} \omega^{2}\right)}\right]
$$

where the following obtain:

$$
\begin{array}{lc}
\bar{\epsilon}=\epsilon_{r}+i \epsilon_{i}, & \Omega_{1}^{2}=\left(\Omega_{+}^{2}-\omega^{2}\right)^{2}, \\
\Omega_{2}^{2}=\left(\Omega_{-}^{2}-\omega^{2}\right)^{2}, & (\hat{\mu} \cdot \hat{\mathrm{e}})^{2}=P .
\end{array}
$$

These expressions may be used to calculate the unmodulated crystal reflection spectrum and the Seraphin coefficients $\alpha$ and $\beta$. Calculation of the piezoreflection spectrum requires the evaluation of $E q$. (3.1). The differential changes in $\bar{\epsilon}$ have the following form:

$$
\begin{aligned}
d \epsilon_{r}= & \epsilon_{0}\left\{\left[\frac{\omega_{0}^{2} P}{\left(\Omega_{1}^{2}+\gamma_{+}^{2} \omega^{2}\right)}\right]\left[\frac{-2 F_{+} \Omega_{1}^{2}}{\left(\Omega_{1}^{2}+\gamma_{+}^{2} \omega^{2}\right)} d \Omega_{+}^{2}+F_{+} d \Omega_{+}^{2}+\Omega_{1} d F_{+}\right]\right. \\
& \left.+\left[\frac{\omega_{0}^{2} P}{\left(\Omega_{2}^{2}+\gamma_{-}^{2} \omega^{2}\right)}\right]\left[\frac{-2 F_{-} \Omega_{2}^{2}}{\left(\Omega_{2}^{2}+\gamma_{-}^{2} \omega^{2}\right)} d \Omega_{-}^{2}+F_{-} d \Omega_{-}^{2}+\Omega_{2} d F_{-}\right]\right\}+\left(\epsilon_{0}-\epsilon_{+}\right)\left(\frac{d v_{0}}{v_{0}}\right)+d \epsilon_{0}, \\
d \epsilon_{i}= & \epsilon_{0}\left\{\left[\frac{\gamma_{+} \omega \omega_{0}^{2} P}{\left(\Omega_{1}^{2}+\gamma_{+}^{2} \omega^{2}\right)}\right]\left[\frac{-2 F_{+} \Omega_{1}}{\left(\Omega_{1}^{2}+\gamma_{+}^{2} \omega^{2}\right)} d \Omega_{+}^{2}+d F_{+}\right]+\left[\frac{\gamma-\omega \omega_{0}^{2} P}{\left(\Omega_{2}^{2}+\gamma_{-}^{2} \omega^{2}\right)}\right]\left[\frac{-2 F_{-} \Omega_{2}}{\left(\Omega_{2}^{2}+\gamma_{-}^{2} \omega^{2}\right)} d \Omega_{-}^{2}+d F_{-}\right]\right\}-\left(\epsilon_{i}\right)\left(\frac{d v_{0}}{v_{0}}\right),
\end{aligned}
$$

where

$$
\begin{aligned}
d \Omega_{ \pm}^{2}= & {\left[\left\{1 \pm\left[\omega_{1}^{2}-\omega_{2}^{2}+\omega_{0}^{2} f_{1} \mathbf{T}(\mathbf{k})-\omega_{0}^{2} f_{2} \mathbf{T}(\mathbf{k})\right] \Delta^{-1}\right\} \omega_{1} d \omega_{1}+\left\{1 \pm\left[\omega_{2}^{2}-\omega_{1}^{2}+\omega_{0}^{2} f_{2} \mathbf{T}(\mathbf{k})-\omega_{0}^{2} f_{1} \mathbf{T}(\mathbf{k})\right] \Delta^{-1}\right\} \omega_{2} d \omega_{2}\right.} \\
& \left.-\left(\left(f_{1}+f_{2}\right) \pm\left\{\left(\omega_{2}^{2}-\omega_{1}^{2}\right)\left(f_{2}-f_{1}\right)+\left[\omega_{0}^{2} f_{1} \mathbf{T}(\mathbf{k})+\omega_{0}^{2} f_{2} \mathbf{T}(\mathbf{k})\right]\left(f_{1}+f_{2}\right)\right\} \Delta^{-1}\right)\left[\left(\frac{\omega_{0}^{2} \mathbf{T}(\mathbf{k})}{2}\right)\left(\frac{d v_{0}}{v_{0}}+\frac{d \epsilon_{0}}{\epsilon_{0}}\right)\right]\right] \\
d F_{ \pm}= & \left(\left[\frac{4 \omega_{1}\left(\Omega_{ \pm}^{2}-\omega_{1}^{2}\right)}{N}\right]\left\{\left[F_{ \pm} \omega_{0}^{4} \mathbf{T}^{2}(\mathbf{k}) f_{2}\right]-\left(\Omega_{ \pm}^{2}-\omega_{2}^{2}\right)^{2}\right\} d \omega_{1}+\left[\frac{4 \omega_{2}\left(\Omega_{ \pm}^{2}-\omega_{2}^{2}\right)}{N}\right]\left\{F_{ \pm} \omega_{0}^{4} \mathbf{T}^{2}(\mathbf{k}) f_{1}\right]-\left(\Omega_{ \pm}^{2}-\omega_{1}^{2}\right)^{2}\right\} d \omega_{2} \\
& +\frac{2}{N}\left[\left(\Omega_{ \pm}^{2}-\omega_{1}^{2}\right)^{2}\left(\Omega_{ \pm}^{2}-\omega_{2}^{2}\right)+\left(\Omega_{ \pm}^{2}-\omega_{2}^{2}\right)^{2}\left(\Omega_{ \pm}^{2}-\omega_{1}^{2}\right)^{2}-F_{ \pm} \omega_{0}^{4} \mathbf{T}^{2}(\mathbf{k}) f_{1}\left(\Omega_{ \pm}^{2}-\omega_{2}^{2}\right)-F_{ \pm} \omega_{0}^{4} \mathbf{T}^{2}(\mathbf{k}) f_{2}\left(\Omega_{ \pm}^{2}-\omega_{1}^{2}\right)\right] d \Omega_{ \pm}^{2} \\
& \left.+\left[2 F_{ \pm}\left(\frac{d v_{0}}{v_{0}}+\frac{d \epsilon_{0}}{\epsilon_{0}}\right)\right]\right)
\end{aligned}
$$

and

$$
N=\left[\left(\Omega_{ \pm}^{2}-\omega_{2}^{2}\right)^{2} \omega_{0}^{4} \mathbf{T}^{2}(\mathbf{k}) f_{1}+\left(\Omega_{ \pm}^{2}-\omega_{1}^{2}\right)^{2} \omega_{0}^{4} \mathbf{T}^{2}(\mathbf{k}) f_{\mathbf{2}}\right] .
$$

${ }^{1} \mathrm{H}$, G. Drickamer and C. W. Frank, Electronic Transitions and the High Pressure Chemistry and Physics of Solids (Chapman and Hall, London, 1973).

${ }^{2}$ S. D. Colson, J. Chem. Phys. 45, 4746 (1966).

${ }^{3}$ D. M. Hanson, J. Chem. Phys. 52, 3409 (1970).

${ }^{4} \mathrm{~J}$. Ferguson, Mol. Cryst. Liq. Cryst. 58, 55 (1980).

${ }^{5}$ D. P. Craig. and S. H. Walmsley, Excitons in Molecular Crystals (Benjamin, New York, 1968).

${ }^{6}$ G. C. Morris, S. A. Rice, and A. E. Martin, J. Chem. Phys. $52,5149(1970)$.

${ }^{7}$ G. C. Morris and M. G. Sceats, Chem. Phys. 1, 376 (1973).

${ }^{8}$ (a) G. C. Morris and M. G. Sceats, Chem. Phys. 3, 164

(1974); (b) J. Chem. Phys. 60, 375 (1974).

${ }^{9}$ R. Mason, Acta Crystallogr. 17, 547 (1964).

${ }^{10}$ P. W. Bridgman, Proc. Natl. Acad. Sci. U.S. A. 76, 9, 71 (1945); 76, 1, 19 (1945); 76, 3, 83 (1948).

${ }^{11} \mathrm{~J}$. Kalinowski, J. Godlewski, and R. Jankowiak, Chem. Phys. Lett. 43, 127 (1976).

${ }^{12}$ (a) B. O. Seraphin and R. B. Hess, Phys. Rev. Lett. 14, 138 (1965); (b) W. E. Engeler, H. Fritzsche, M. Garfinkel, and J. J. Tiemann, Phys. Rev. Lett. 14, 1069 (1965); (c) G. W. Gobeli and E. O. Kane, Phys. Rev. Lett. 15, 142 (1965); (d) M. Garfinkel, J. J. Tiemann, and W. E. Engeler, Phys. Rev. 148,695 (1966).
${ }^{13}$ (a) B. Batz, Solid State Commun. 4, 241 (1966); (b) I. Balslev, Phys. Rev. 143, 636 (1966).

${ }^{14}$ (a) M. Cardona, Modulation Spectroscopy, in Solid State Physics, Supplement 11, edited by H. Ehrenreich, F. Seitz, and D. Turnbull (Academic, New York, 1969); (b) Proceedings of the First Intemational Conference on Modulation Spectroscopy, edited by B. O. Seraphin (North-Holland, Amsterdam, 1973); (c) "Modulation Techniques," in Semiconductors and Semimetals, edited by R. K. Willardson and A. C. Beer (Academic, New York, 1972), Vol. 9.

${ }^{15}$ R. M. Hochstrasser and L. J. Noe, Chem. Phys. Lett. 5, 489 (1970).

${ }^{16}$ A. Matsui, K. Tomioka, Y. Oeda, and T. Tomotika, Surf. Sci. 37, 849 (1973).

${ }^{17}$ P. I. Perov and J. E. Fischer, Phys. Rev. Lett. 33, 521 (1974).

${ }^{18}$ M. A. E1-Sayed, Acc. Chem. Res. 4, 23 (1971).

${ }^{19} \mathrm{C}$. J. Eckhardt and J. Merski, Surf. Sci. 37, 937 (1973).

${ }^{20}$ B. M. Fanconi, G. A. Gerhold, and W. T. Simpson, Mol. Cryst. Liq. Cryst. 6, 41 (1969).

${ }^{21}$ J. Merski and C. J. Eckhardt (to be published).

${ }^{22}$ B. O. Seraphin, in Optical Properties of Solids, edited by F. Abeles (North-Holland, Amsterdam, 1972), p. 167.

${ }^{23}$ D. D. Sell and E. O. Kane, Phys. Rev. 185, 1103 (1969). 
${ }^{24}$ B. O. Seraphin and N. Bottka, Phys, Rev. 145, 628 (1966).

${ }^{25}$ S. A. Rice and J. Jortner, in Physics of Solids at High Pressures, edited by C. T. Tomizaka and R. M. Emrick (Academic, New York, 1965), p. 63.

${ }^{26} \mathrm{H}$. C. Longuet-Higgins and J. A. Pople, J. Chem. Phys. 27, 192 (1957).

${ }^{27} \mathrm{~L}$. Onsager, J. Am. Chem. Soc. 58, 1486 (1936).

${ }^{28}$ N. S. Bayliss, J. Chem. Phys. 18, $292(1950)$.

${ }^{29}$ A. J. Davydov, Theory of Molecular Excitons (Plenum, New York, 1971); R. M. Hochstrasser and P. N. Prasad, J. Chem. Phys. 56, 2814 (1972).

${ }^{30}$ P. E. Schipper, Mol. Cryst. Liq. Cryst. 28, 401 (1974).

${ }^{31}$ M. R. Philpott, in Advances in Chemical Physics, edited by

I. Prigogine and S. A. Rice (Academic, New York, 1973),
Vol. 23.

${ }^{32}$ G. D. Mahan, J. Chem. Phys. 41, 2930 (1964); 43, 1569 (1965).

${ }^{33}$ R. R. Pennelly and C. J. Eckhardt; Chem. Phys, 12, 89 (1975).

${ }^{34}$ J. J. Hopfield, J. Phys. Soc. Jpn. 21, 77 (1966).

${ }^{35}$ J. Merski and C. J. Eckhardt, Chem. Phys. Lett. 63, 174 (1979).

${ }^{36}$ C. J. Eckhardt, R. J. Hood, J. Merski, and J. Tylicki, J. Lumin. 12, 805 (1976).

${ }^{37}$ G. E. Hardy, J. C. Baldwin, J. I. Zink, W. C. Kaska, P. H. Liu, and L. Dubois, J. Am. Chem. Soc. 98, 3552 (1977). ${ }^{38}$ M. Cohen, Z. Ludmer, J. M. Thomas, and J. O. Williams, Proc. R. Soc. London Ser. A 324, 459 (1971). 